\title{
Bibliografía del Doctor Julio C. Tello
}

A.-Bibliografia: irabajos publicados.

B.-Bibliografía: trabajos inédilos.

C.-Necrología - Homenajes.

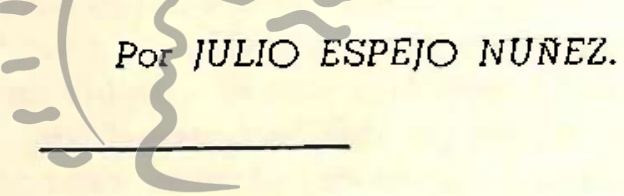

\section{A.-BIBLIOGRAFIA, i Trabajos publicados etras "Jorge Puccinelli Converso" \\ INDICE CRONOLOGICO}

1905 Craniectomía Prehistórica entre los Yauyos, Conferencia. "El Comercio", Lima, 5 de Mayo.

908 La antíguedad de la síflis en el Perú. Revista Universitaria. Lima. La misma obra publicada en San Martí y Cía. po. 22, XV láminas. Lima, 1909.

1909 Consideraciones scbre la monografía "La uta en el Perú" por Manuel $O$. Tamayo. (En colaboración con el Dr. Ricardo Palma).

1912 Prehistoric trephining among the Yauyos of Peru. XVII International Congress of Americ. pp. 75-83. London.

1913 Presente y futuro del Museo Nacional. Lima.

1913 Algunas conexiones gramaticales de las lenguas Campa, Ypurina, Moxo, Amuesh, Goajira, del grupo á familia Arawak ó Maipuru. Revista Universitaria, pp. 506-532, Año VII, Vol. I. Limá. 
1913 La ciencia antropoiógica en el Perú. "La Prensa", Lima, 23 de Mayo.

1913 Patclogía y prácticas quirúrgicas entre los antiguos peruanos. "Congreso Médico Americano", Lima, Noviembre.

:913 Arawak, fragmento de linguística indígena Sudamericana. Lima.

1914 Las supuestas maravillas del Valle del Rímac. "La Crónica", Lima, Diciembre.

1914 Las antiguas riquezas del Valle de Lima. "La Crónica", Lima, 30 de Diciembre.

1915 El curioso final de una polémica arqueológica. "La Crónica", Lima, 3 de Enero.

1915 El uso de las cabezas humanas artificialmente momificadas. El Comercio. Lima, 29 de agosto.

1915 El diagnóstico diferencial entre las aberturas craneales por trepa ción y las practicadas en las cabezas trofeo. "Sociedad Médica Unión Fernandina". Lima, Octubre.

1917 Los antiguos Cementerios del Valle de Nasca. Washington.

1918 El uso de las cabezas humanas artificialmente momificadas y su representación en el Antiguo Arte Peruano. Revista Universitaria. Año XIII. Vol. III. Pp. 477-533. Lima.

1918 Arqueología y primitiva religión del Perú. La Prensa, Lima, 11 de julio.

1919 Folk-Lore Indiano: Leyenda de la génesis deSlos Amueshas. Revista cie Psiquidiníag disciplinașliconexaserTomo I, pp. 51-55. Lima.

is 19 La necesidad de reconstruir la historia de la Civilización y de la Cultura Patria remontándose hasta su origen. Conferencia en el Teatro Rivera de Huaraz, marzo, "El Departamento", 19 de abril.

1919 Tello en la Universidad. "Variedades", Año XV, No 591, Lima, 28 de Junio.

1920 Cultura Arcaica, Cultura Media y Cultura Alta. Prólogo a la obra "Los Incas del Perú" por Clemente R. Markham. Versión castellana de Manuel Be!troy. Lima.

:921 La renuncia del Director del Museo Nacional de Arqueología Dr. Philip A. Means. (Declaración). El Comercio, 11 de marzo.

1921 Estudios antropológicos en el Departamento de Ancash. Archivos de la Asociación Peruana para el progreso de la ciencia. Tomo L pp. 131-137. Lima.

1921 Introducción a la Historia Antigua del Perú. Editorial Euforion. Pp. 48, XXVII láminas. Lima. 
1921 Sobre un proyecto del Museo Incaico. "El Comercio", Lima, Noviembre.

1022 Prehistoric Peru. Inter-America, pp. 238-250. New York.

1922 La investigacićn científica. Discurso de orden pronunciado en la sesión inaugural de la Asociación Peruana para el Progreso de la Ciencia, 30 de Julio. Sanmartí y Cía. Lima.

1923 Wira-Kocha. Inca, Vol. I, No 1, pp. 94-320 y Vol. I, No 3, pp 583606. Lirna.

1923 Wallallo. Ceremonias gentílicas realizadas en la región Cisandina del Perú Central, Distrito arqueológico de Casta. (En colaboración de Próspero Miranda). Inca. Vol. I, N? 2, pp. 475-549. Lima.

1923 Ortografía fonética de las lenguas indígenas. Sistema de sonidos de la Kesnwa. Inca Vol. I, No 2, pp. 550-556. Lima.

1923 Observaciones al discurso del Profesor Seler. Inca. Vol I, No 2, pp. 375-382. Lima.

1923 Folk-Lore Anoino: Tiula-Kamake (Texto Aimara), Huachuahuan Atok (Texto Wanka), Atoxwan Wachwan (Texto Chinchaysuyo). En colaboración de Adolfo Vienrich. Inca. Vol I, No.2, pp. 421-431. Lima.

1924. Sobre la autenticidad de unos huacos. (Declaración). El Comercio, Lilma, 13 de Junio.

1924 El Museo Arqueológico Nacional. Conferencia. El Comercio, Lima. 14 de Diciembre.

1925 El Museo de Arqueología Peruana: sus fines y propósitos. Lima.

1926 Interesantes descubrimientos arqeuológicos en Cerro Colorado. Paracas. El Comercio. Lima, 6 de Febrero.

1926 I.os descubrimientos del Museo de Arqueología Peruana en la Península de Paracas. La Prensa. Lima, 7 y 8 de Junio.

1927 Bibliografía Antropológica del Perú. Boletín Bibliográfico, publicado por la Universidad Mayor de San Marcos. Vol. III, No 3, pp. 31-36. Lima.

1928 Campo neutral. Las acusaciones de Dn. Victor Larco Herrera a Dn. Julio C. Tello. Carta. La Prensa. Lima, 18 de Mayo.

1928 Nota comentario a la Obra "Coricancha" de R. Lehmam-Nitsch. En Boletín Bibliográfico. Año VI, Vol. III, No 4, pp. 201-209. Lima.

1928 Reforma Universitaria. Ensayos y Discursos. Sanmartí y Cía. Lima.

1928 Andean Civilization: Some problems of peruviam Archaeology. XXIII International Congress of Americanists. New York. 
1928 Los descubrimientos del Museo de Arqueología Peruana en la Península de Paracas. International Congress of Americanists, XXII, Pt. 1, pp. 679-690. Roma.

1929 Antiguo Perú. Primera Edad. Editado por la Comisión organizadora del Segundo Congreso Sudamericano de Turismo. Emp. Ed. Excelsior Pp. 183. Lima.

1929 La indumentaria de los Incas. En "Letras". Año I. Vol. II, pp. 4l3419. Lima.

1929 Museo de Arqueología Peruana: Exposición especial de Arte Antiguo Peruano. (Conferencia). El Comercio. Lima, 17 de Octubre.

1930 An Ancient Syphilitic Skull from Paracas in Peru. (En colaboración de Williams Herbert). Annals of Medical History, Vol. 2, N? 5, pp. 515-529, con ilustraciones. New York.

1930 Del periódico, del Editor, de los títulos de los artículos y de los autores de éstos; de las acusaciones propiamente dichas; del desprestigio que éllas pretenden producir, atacando injustificadamente a personas e instituciones. (Carta). El Comercio. Lima, 16 de Setiembre.

1931 Ruinas de Wari en Ayacucho. El Perú. Lima, 27 de Agosto.

1931 Prólogo. Revista "Wira-Kocha". Vol. I, No 1., pp. l-3. Lima.

1931 Sistema fonético de las lenguas indígenas del Perú. Wira-Kocha. Vol. I, No 1, pp. 4-8. Lima.

1931 Un modelo de escenografía plástica en el Arte Antiguo Peruano. Wira Kocha. Vol. I,oNgelPpp. $89-112$. Lima.rerso»

1931 Instituto Nacional de Antropología. Wira-Kocha. Vol. I., No 2, pp. 117-124. Lima.

1932 La defensa de nuestros tesoros arqueológicos: la importancia del yacimiento arqueológico de Paracas, la destrucción de antigüedades y de las medidas adoptadas para su conservación. El Comercio. Lima, 24 de mayo.

1932 La defensa de nuestros tesoros arqueológicos: el importante yacimiento arqueológico de Paracas y su destrucción. La Industria. Piura, 3 de Junio.

1933 Nepeña. La Crónica. Lima, 8 de Setiembre.

1933 Descubrimiento arqueológico en el Valle de Nepeña. El Comercio. Lima, 10 de Setiembre.

1933 Notable monumento arqueológico en el Valle de Nepeña (Carta a' Ministro de Instrucción Pública). La Crónica. Lima, 11 de Setiembre. 
1933 Sensacional y valioso hallazgo de carácter científico en el Valle de Nepeña (Declaraciones). El Comercio. Lima, 24 de Setiembre.

1933 El Palacio de Cerro Blanco, en Nepeña. (Declaraciones). El Comercio. Lima, 3 de Octubre.

1933 Las ruinas del Valle de Nepeña. (Declaraciones). El Comercio. Lima, 5 de Octubre.

1933 Una Civilización superior a todas las Civilizaciones Precolombinas cree encontrar el Doctor Tello en el Valle de Nepeña. (Declaraciones). La Crónica. Lima, 5 de Octubre.

1933 Agasajo al Doctor Julio C. Tello (Conferencia). El Comercio. Lima, 27 de Octubre.

1934 El oro en el Ãntiguo Perú. El Comercio. Lima, 1\% de Enero.

1934 Excavaciones arqueológicas en el Cuzco. El Comercio. Lima, 12 y 15 de Marzo. El Liberal. Lima, 5 de: Marzo.

1934 Perú Prehistórico: Origen desarrollo y Correlación de las Antiguas Culturas Peruanas. Revista de la Universidad Católica del Perú. Tomo II, N? 10, pp. 151-168. Lima.

1935 Las Civilizaciones Pre-Inkaicas, su antigüedad y sucesión cronológica. (Conferencia). La Crónica. Lima, 30 de Mayo.

1935 Culturas Arcaicas del Horizonte inferior. (Conferencia). Universal. Lima, 13 de Junio.

1935 Varios aspectos interesantes de la Civilización Incaica. (Conferencia). Universal. Lima, 19 Qde Zagosto. Letras

1935 ¿Barbarie ó Givilización? Eh Comercio Lima 19 de Octubre.

1935 Prólogo a la obra "Las Culturas Pre-hispánicas del Departamento de Lima" por Eduardo Villar Córdova. Lima.

1936 Indumentaria de los Inkas. Letras, № 2, pp. 413-419. Lima. . El. Comercio. Lima, 29, 30 y 31 de Enero.

1936 Los monumentos arqueológicos de Magdalena Vieja y la necesidad de conservarlos. Gaceta Municipal. Magdalena Vieja, Enero.

1936 Las Civilizaciones Pre-Incaicas del Perú. Conferencia. En "PostBulletin", Rochester, Minnesota, Friday, September 25. El Comercio. Lima, 25 de Octubre.

1936 Dioses y Demonios del Antiguo Perú. (Conferencia en la Academia Nacional de Ciencias "Antonio Alzate"). El Universal, México, 8 de Noviembre. La Prensa, Lima, 22 de Noviembre.

1936 Prólogo a la obra "Del Ayllu al cooperativismo Socialista" de Hi1debrando Costro Pozo. Lima.

1937 Los trabajos arqueológicos en el Departamento de Lambayeque. El Comercio, Lima, 29, 30 y 31 de Enero. 
1937 La búsqueda de tesoros ocultos en las huacas de Lambayeque. El Comercio, Lima, 11 de Marzo.

1937 El Oro de Batán Grande. El Comercio. Lima, 18 de Abril.

1937 Importante hallazgo arqueológico en la Huaca, Cerro Sechín, de Casma. El Comercio. Lima, 28 de Setiembre.

1937 Objeto y propósito de la Expedición Arqueológica al Marañón. El Comercio. Lima, 31 de Octubre. También en el "Boletín de la Sociedad Geográfica" Tomo LV. Lima.

1937 Significado de Ayllu. Nota editorial de la revista "Ayllu", Organo del Instituto de Sociología Peruana. Año l, No l. Lima.

1937 La Civilización de los Inkas. Letras. Tomo III, No 6, pp. 5-37. Lima.

1937 El Strombus en el Arte Chavín. El Comercio. Lima, 18 de Abril. - Turismo. Lima, Agosto, 1938.

1938 Los resultados de la Expedición Arqueológica al Marañón de 1937. El Comercio, Lima, 9 de Enero.

1938 Las excavaciones que se efectúan en Pachacamac. El Comercio. Lima, 13 de Setiembre.

1938 La Gran Muralla del Norte del Perú: es un camino de penetración de la Costa a la Sierra, de carácter comercial y principalmente religioso. El Comercio. Lima, 11 de Octubre.

1938 Una notable insignia de oro del Antiguo Perú. Turismo. № 133. Lima, mes de Noviembre.

1938 Arte Antiguo Peruano; Album fotográfico de las principales especies arqueológicas existentes en los Museos de Lima. Primera Parte: Tecnología y Morfología. Inca Vol. II. Lima.

1938 Museo de Antropología: Exposición especial de Arte Aborigen realizada en honor a los delegados de la VIII Conferencia Internacional Americana. "Editorial D. Miranda". Lima.

1939 El Cóndor en el Arte Antiguo Peruano. Turismo. Lima, mes de Mayo.

1939 Algunos monumentos arqueológicos existentes entre Lima y Paramonga. El Comercio. Lima, 27 de Setiembre.

1939 The Empire of the Inkas. So Live the works of Men. Seventieh Anniversary Volume Honoring Edgard Lee Hewett. Alburquerque.

1939 Need to Establish an International Institute of Archaeology. American Scientific Congress. Washington, D. C.

1939 Las Primeras Edades del Perú por Guaman Poma. Ensayo de interpretación. Empresa Gráfica T. Scheuch. Lima. 
1939 Estatuto de la Asociación Peruana de Arqueología. Lima, Octubre.

1940 El Descubrimiento de Esculturas Monolíticas en la Waka Cerro Sechín, Valle de Casma. La Prensa. Lima, 7 de Enero.

1940 Descubrimientos realizados en las ruinas de Pachacamac. El Comercio, Lima, 18 de Julio.

1940 Los recientes descubrimientos arqueológicos en las ruinas de $\mathrm{Pa}$ chacamac. Turismo. Lima, mes de Julio.

1940 Pachacamac. El Comercio. Lima, 4 de agosto.

1940 Prólogo a la Revista Chaski. Organo de la Asociación Peruana de Arqueología. Vol. I. N? 1. Lima.

1940 Un vaso de piedra de Nasca. Primeros indicios de una Cultura megalítica semejante a la de Chavín en la región Central del $\mathrm{Pe}$ rú. Chaski. Vol. I, No 1. pp. 27-48. Lima.

1940 Pachacamac. Chaski. Vol. I, No 2. pp. 1-4. Lima.

1940 Un ejemplar de cerámica de Cajabamba. Chaski. Vol. I, N² 2. pp. 77. Lima.

1940 Comentario a la obra "Verruga Peruana" por Raúl Rebagliati. Chaski. Vol I, No 2. pp. 87-88. Lima.

1940 El lugar que ocupa la ciencia arqueológica en el campo del conocimiento humano. (Conferencia). Chaski. Vol. I, No 1, pp. 84-85. Lima.

1941 La creación de Parques Históricos Nacionales. El Comercio. Lima, 29 de Enero.

1941 El valor nacionalista de la Arqueología. El Comercio. Lima.

1941 Hacia el Perú Jen gos Pdeloro del los Inkas.sBoletín de la Comisión Nacional Peruana de Cooperación intelectual. Primer Trimestre, pp. 57-61. Lima. - La Razón, Cajamarca, 12 de Octubre. Universidad, No 2, Lima, 1947.

1941 La Ciudad Incaica de Cajamarca. Chaski .Vol. 1, No 3, pp. 3-7. Lima.

1941 El Museo de Antropología e Instituto de Investigaciones Antropológicas. Chaski. Vol. I, No 3, pp. 72-78. Lima.

1942 Conferencia en la Universidad del Cuzco, 2 de Noviembre.

1942 Importancia arqueológica e histórica de la piedra Saiwile. Conferencia en el Coleyio Nacional de Mujeres de Abancay. 8 de Noviembre.

1942 Aporte de los Estados Unidos a la Arqueología Peruana. En "Peruanidad". Vol II, N? 6, pp. 483-485. Lima. 
1942 Origen y desarrollo de las Civilizaciones Prehistóricas Andinas. "Actas y trabajos científicos del XXVII ${ }^{\circ}$ Congreso Internacional de Americanistas, Lima, 1939". Tomo I, pp. 589-720. Lima, Perú, 1940. - Reimpreso en Librería é Imprenta Gil, S. A., pp. 132, XXIII láminas. Lima.

1943 Memoria suscinta sobre los trabajos arqueológicos realizados en las ruinas de Pachacamac durante los años 1940 y 1941. En "Memoria de la Junta Departamental Pro-Desocupados de Lima', 1939, 1940 y 1941 ". Lima.

1943 Discovery of the Chavin Culture in Peru. Reprinted from "American Antiquity", Volume 9, № 1, XXIV láminas. July.

1943 Sobre el descubrimiento de la Cultura de Chavin en el Perú. Letras. Tercer Cuatrimestre. Pp. 326-373. Lima. -Librería Gil, S. A., Pp. 1-54. VII láminas. Lima, 1944.

1943 To Peru in Quest of Inka.Gold. "América Indígena", Vol. III. pp. 115-126. México.

1944 Militancia de la Arqueología. (Declaración). "Turismo", Año IX, No 94. Lima, mes de Abril.

1944 América puede llegar a ser un país de Indios... (Declaraciones). En "Excelsior", México, 28 de Enero.

1944 La falsificación como síntoma de una Cultuia descendente. La Prensa. Lima, 28 de Febrero.

1944 Aporte de los Estados Unidos a la Arqueología Peruana. "El Callao", Callao, 26 de Diciembre.

1944 Militancia de la Arqueología. (Declaración). "Turismo", Año IX, No 94. Lima, mes de Abril.

1944 Prólogo al opúsculo "Peculiaridades anatómicas en los cráneos paleoperianos" por Ricardo Palma. Editorial Lúmen. S. A. Lima.

1945 La primera piedra del edificio del Museo Nacional de Antropología y Arqueología. La Crónica. Lima, 27 de Julio.

1945 El país de los Inkas. En "Perú en Cifras 1944-45". Empresa Gráfica Scheuch S. A., pp. 592-613, XX ilustraciones. Lima.

1946 Los tesoros arqueológicos de Ancón. El Comercio, Lima, 17 de Marzo.

1947 Sobre el descubrimiento de "Una Antigua Ciudad Planificada" en el Valle del Virú por una Expedición Científica de los Estados Unidos. (Declaraciones). La Prensa. Lima, 16 de Febrero. 
B.-BIBLIOGRAFIA: trabajos inéditos

(Indice del Archivo legado a la Universidad Nacional Mayor de San Marcos)

1.-Expedición arqueológica al Marañón, 1937.

2.-Monografía "Pachacamac".

3.-Arqueología del Valle de Lima.

4.-Expedición arqueológica a la Huaca Malena, Valle de Asia, 1925.

5.-Monografía "Kipu".

6.-Monografía sobre lingüística peruana: Akaro, Quechua y Aimara

7.-Expedición arqueológica de 1919.

8.-Expedición arqueológica al Valle de Nepeña.

9.-Monografía "Muchik".

10.--Monografía "Chavin".

11.--Monografía "Inka".

12.-Monografía "Chimú".

13.-Monografía "Chanka".

14.--Monografía "Mantaro".

15.-Monografía "Paracas".

16.-Monografía "Tiahuanaco".

17.-Monografía "Nasca".

18.-Monografía de Huánuco Viejo. Le Letras

19.-Monografía déTambolColorado. Converso»

20.-Análisis de los Cronistas del Perú .

21.-Traducciones de obras antropológicas.

C.-NECROLOGIAS - HOMENAJES

(Indice cronológico)

1947 - Ayer falleció el Arqueólogo Dr. Julio C. Tello. La Tribuna. Lima, 4 de Junio.

-Falleciniento del Dr. Julio C. Tello. El Comercio. Lima, 4 de Junio. (Edición de la mañana y de la tarde). 
- Actualidad: Julio C. Tello. El Comercio, edición de la tarde. Lima, 4 de Junio.

-Falleció ayer el Dr. Julio C. Tello. La Crónica. Lima, 4 de Junio.

-Falleció ayer el Dr. Julio C. Tello. La Prensa. Lima, 4 de Junio.

-Duelo en las Instituciones científicas del Perú por la muerte del Arqueólogo Dr. Julio C .Tello. Le rendirán honores de Ministro de Estado. La Tribuna. Lima, 5 de Junio.

-Julio C. Tello, Amauta Preclaro. La Tribuna. Lima, 5 de Junio.

- Mañana se realizarán los funerales del eminente arqueólogo Julio C. Tello. El Comercio. Lima, 5 de Junio.

- Homenaje a la memoria de Julio C. Tello por William Benton y William Duncan Strong. El Comercio. Lima, 5 de junio.

- Mañana serán inhumados los restos del que fuera Dr. Julio C. Tello. La Prensa. Lima, 5 de junio.

-Repercusión del fallecimiento del Dr. Julio C. Tello. La Prensa. Lima, 5 de junio.

-Relámpagos: Julio C. Tello. La Prensa. Lima, 5 de junio. .

- Honores de Ministro de Estado se tributará a los restos del Doctor Tello. La Crónica. Lima, 5 de junio.

-Julio C. Tello. "El Callao". Callao, 5 de junio.

--Hoy serán inhumados los restos del Dr. Tello a quien se le rendirán honores de Ministro. La Tribuna. Lima, 6 de junio.

- Primer plano: Tello y el eterno Perú. La Tribuna. Lima, 6 de junio.

- Hoy se efectuará el sepelio del sabio peruano Dr. Julio C. Tello. La Prensa. Lima, 6 de junio.

- En la mañana de hoy serán. sepultados los restos del Dr. Julio C. Tello. El Comercio. Lima, 6 de junio.

- Homenaje a la memoria del Dr. Tello por el Secretario del Smithsonian Institute, Alexander Wetmore. El Comercio. Lima, 6 de junio.

- Sentida y elocuente manifestación constituyó el sepelio del Dr. Tello. El Comercio. Edición de la tarde. Lima, 6 de junio.

- En la mañana de hoy efectuánse los funerales del Dr. Tello. La Crónica. Lima, 6 de junio.

- Mi homenaje al arqueólogo, peruano Julio C. Tello, Director del Museo de Antropología, por José María Morante, fallecido ayer en Lima. El Deber. Arequipa, 6 de Junio. 
- Con honores de Ministro de Estado fueron sepultados ayer provisoriamente en el Cementerio General los restos del ilustre arqueólogo Dr. Julio C. Tello. Su cadáver descansará definitivamente en el Museo Antropológico que él creó y organizó. La Tribuna. Lima, 7 de junio.

- Homenaje de "La Tribuna" al sabio Tello. La Tribuna. Lima, 7 de junio.

-Conservemos y prosigamos la obra de Tello. La Tribuna. Lima, 7 de junio.

-El sepelio del Dr. Tello constituyó una imponente manifestación de duelo. El Comercio. Lima, 7 de junio.

- Sentida manifestación de duelo constituyó ayer el sepelio de los restos del Dr. Julio C. Tello. La Crónica. Lima, 7 de junio.

-Una cuartilla: Julio C. Tello. Jornada. Lima, 7 de.junio.

-Fueron trasladados al Cementerio General los restos del Doctor Julio C. Tello. La Prensa. Lima, 7 de junio.

-Condolencia del Institute of Andean Research por la muerte del Dr. Julio C. Tello. El Comercio. Lima, 8 de junio.

- Manifestación de pesar por la muerte del Dr. Tello en Chavin de Huantar. El Comercio. Lima, 10 de junio.

- Se designa con el nombre de Julio C. Tello, el Colegio Nacional de Varones de Matucana. La Prensa. Lima, 10 de junio.

- Centros de Cultura de América saludan a un peruano ilustre. Vanguardia. Limà, lo de junio.

-El sabio Julio C. Tello y el Áprísmo por Julio César Pozo. La Tribuna. Limá, $\mathrm{H}$ - de junio.

- Condolencia de la Unión Panamericana por la muerte del Dr. Julia C. Tello. La Crónica. Lima, 12 de junio.

-Una vocación de sabio. El Comercio. Edición de la tarde. Lima, 12 de junio.

- Legó el Dr. Tello a la Universidad su Biblioteca, Archivos y Libretas de campo. La Crónica. Lima, 12 de junio.

-Rindieron homenaje a Julio C. Tello, los alumnos del Colegio Nacional de San Marcos. La Tribuna. Lima, 13 de junio.

- Dr. Julio C. Tello. Sierra, Lima, primera quincena de Junio.

-Forman Comité Provincial Huarochirano Pro-Homenaje al sabio Julio C. Tello. La Tribuna. Lima, 19 de junio.

-Rindió homenaje al Dr. Julio C. Tello la Junta Municipal de Huarochiri. La Tribuna. Lima, 28 de junio. 
-Ofrecen su aporte para la Biografía del sabio Tello varios de sus condiscípulos. La Tribuna, Lima, 29 de Junio; El Comercio. Lima, 30 de junio.

-Nuestro homenaje al Doctor Julio C. Tello. La Revista Rosa. Año V. No 16. Lima, 30 de junio.

- Sensible deceso del Dr. Julio C. Tello. Resurgimiento. Edición extraordinaria. Lima, mes de junio.

- Ha muerto uno de los más grandes huarochiranos de nuestra época, por Antonio Flores. Resurgimiento. Lima, mes de junio.

- Relación de grados y honores conferidos al Dr. Julio C. Tello. Resurgimiento. Lima, mes de junio.

-El sabio Antropólogo huarochirano Julio C. Tello. Resurgimiento. Lima, mes de junio.

-Relación de libros y folletos publicados por el Dr. Julio C. Tello. Resurgimiento. Lima, mes de junio.

-Julio C. Tello. "Alerta". Lima, mes de junio.

- Homenaje del Concejo Municipal de Pueblo Libre. La Tribuna. Lima, $1^{\circ}$ de julio.

- Se tributó diversos homenajes a la memoria del Dr. Julio C. Tello. La Tribuna. Lima, 4 de julio.

-Emocionado homenaje rindieron al sabio Julio C. Tello en la Facultad de Medicina. La Tribuna. Lima, 5 de julio.

-Como se miden ciertas vidas, por Marcelino Flores. "Contra-Ataque". Lima, 9 de julio.

- Homenaje en reloConcejo deinla Facultad des Letras, Universidad Mayor de San Marcos. La Tribuna. Lima, 9 de julio.

-Julio C. Tello. En "Colegio". Suplemento de la Revista Universidad. Lima, 11 de julio.

-Nota que dirijieron los redactores de "Universidad", solicitando se rinda homenaje público al eminente hombre de ciencia Julio C. Tello. "Colegio". Lima, 11 de julio.

-En honor del sabio peruano Julio Tello (G. Luna Iglesias y Cristóbal de Losada y Puga). La Prensa. Lima, 13 de julio.

- Se da el nombre de Julio C. Tello a una Escuela de Huarochirí. La Prensa. Lima, 13 de julio.

-Nuestro Museo Arqueológico, por Elvira García y García. El Co. mercio. Lima, 15 de julio.

-Relámpagos: Museos Nacionales. La Prensa. Lima, 20 de julio. 
- Algunos descubrimientos arqueológicos del sabio peruano Doctor Julio C. Tello en el país de los Inkas, por Toribio Mejía Xesspe. El Comercio. Lima, 28 de julio.

-El Museo Nacional de Antropología y Arqueología, por Rébeca Carrión Cachot. La Prensa. Lima, 28 de julio.

-Comité Provincial Huarochirano Pro-Homenaje Julio C. Tello. "Resurgimiento", Lima, Agosto.

- Homenaje a la memoria del Dr. Julio C. Tello, al cumplirse seis meses de su fallecimiento. Facultad de Letras de la Universidad Nacional Mayor de San Marcos. Lima, 3 de Diciembre.

-Un Gran Amauta al Nicho de la Fama. "La Tribuna", Lima, 3 de Diciembre.

- Homenajes Plenos: Julio C. Tello, por Luis Alberto Sánchez. "La Tribuna", Lima, 8 de Diciembre.

- Llevarán el nombre de Julio C. Tello las promociones de los Colegios Nacionales. "La Tribuna", "La Prensa", "El Comercio". Lima, 12 de Diciembre.

1948 -Primera piedra del mausoleo al Dr. I. Tello. "La Tribuna". Lima, 4 de Enero.

-Federico Villareal fué hijo del pueblo; al igual que Julio Tello. "La Tribuna". Lima, 5 de Febrero.

-Homenaje aprista a Julio C. Tello. "La Tribuna". Lima, 12 de Enero.

- Colocarán una placa de bronce en la casa donde nació el arqueólogo Dr. Julio C. Tello. "La .Tribuna". Lima, 29 de Mayo.

-Universitariós rendirán homenaje al sabio Tello. "La Tribuna", Lima, 30 de Mayo.

- Mañana serán trasladados al Museo Arqueológico los restos del Dr. Tello. "La Tribuna", "El Comercio", "La Prensa", Lima, 2 de Junio.

- Hoy serán inhumados los restos del sabio Doctor Julio C. Tello. "La Tribuna", "El Comercio", "La Prensa", "La Crónica". Lima, 3 de Junio.

- La labor arqueológica de Tello. "El Comercio", Lima, 3 de Junio. -Con extraordinario fervor patriótico se inhumaron ayer los restos del sabio peruano Julio C. Tello. "La Tribuna", "La Prensa", "El Comercio", "La Crónica". Lima, 4 de Junio.

-Discurso del señor Rector de la Universidad Nacional Mayor de San Marcos Doctor Luis Alberto Sánchez, con motivo del aniver- 
sario del fallecimiento del Doctor Julio C. Tello. "El Callao", Callao, 5 de Junio.

-J. Tello, paradigma de peruanidad, por Antonio Garland. "La Crónica", Lima, 6 de Junio.

-El Museo de Arqueología y el sabio Julio C. Tello. "La Tribuna". Lima, 6 de Junio.

- Emocionado Homenaje rindió el Congreso Aprista al sabio Tello. "La Tribuna", Lima, 6 de Junio.

-Del ambiente: Falta de Edificio, por Luis B. Miranda. "El Callao", Callao, 10 de Junio.

-El Grupo Waman Poma y el sabio Julio C. Tello. "La Tribuna" y "El Callao". Lima, 15 de Junio.

-Academia del Pueblo "Julio C. Tello". "La Tribuna", Lima, 17 de Junio.

- Recuerdo personal del Dr. Julio C: Tello, por Luis Valle Goicochea. "El Comercio", edición de la tarde, Lima, 23 de Junio.

-En la "Revista del Museo Nacional de Antropología y Arqueología". Vol. II, Primer Semestre No T, Lima, 1948, los siguientes artículos:

-Julio C. Tello y la Arqueología Peruana, por Rebeca Carrión Cachot .

-Apuntes biográficos sobre el doctor Julio C. Tello, por M. Toribio Mejía Xesspe.

-Tello hizo ciencia cơno los Inkas, Pedro Weiss.

- Un recuerdo de Julio C. Tello y Paracas, por Samuel K. Lothrop.

-Julio C. Tello, "Companero y Guía, por William Duncan Strong.

-Julio Tello: erudito y científico peruano, por Watt Stewart.

- Bibliografía del Dr. Julio C. Tello, por. Julio Espejo Núñez.

\section{JULIO ESPEIO NUÑEZ,}

Jefe del Departamento de Exploraciones y Exhibiciones del Museo Nacional de Antropología y Arqueología. 